\title{
Characterizing and Modeling the Apparent Anomalous Behavior of Resistivity in Cr-Si-O Thin Films
}

\author{
Alan F. Jankowski \\ This paper was prepared for submittal to the \\ 25th International Conference on Metallurgical Coatings and Thin Films \\ San Diego, CA \\ April 27 - May 1, 1998
}

March 1998

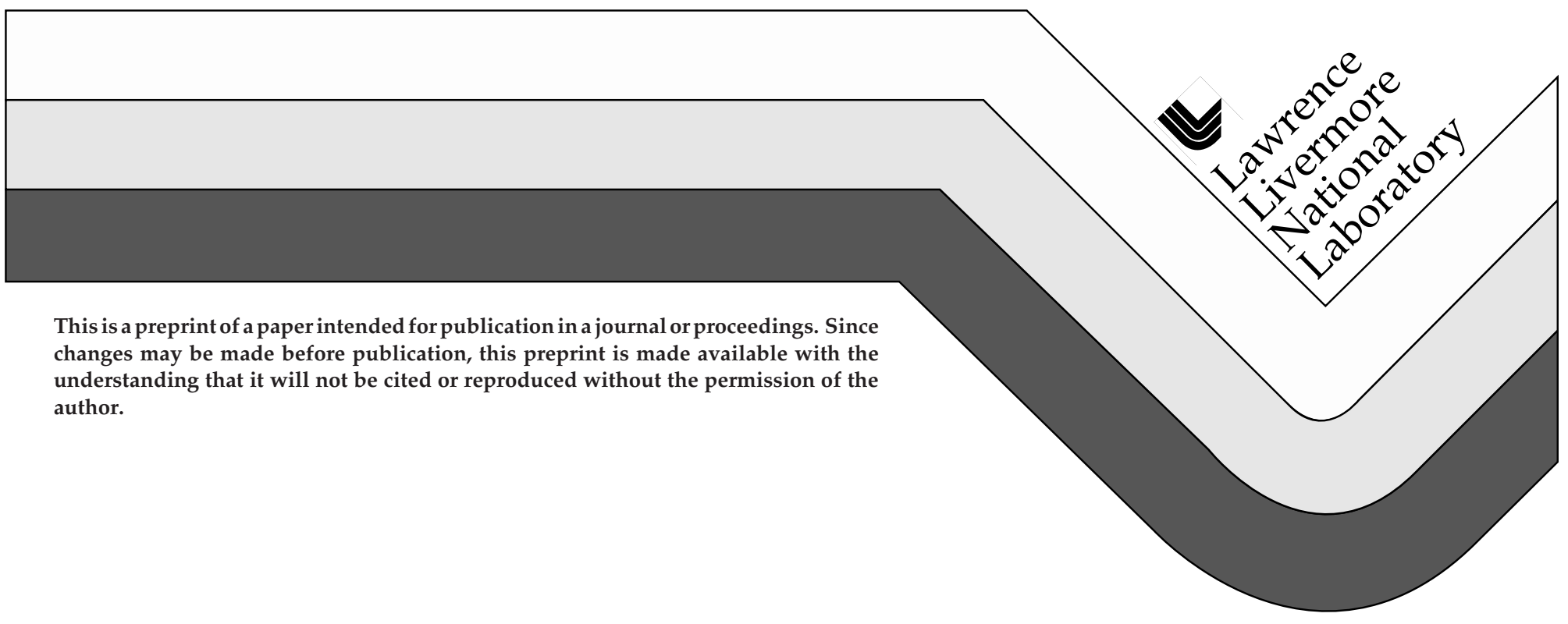




\section{DISCLAIMER}

This document was prepared as an account of work sponsored by an agency of the United States Government. Neither the United States Government nor the University of California nor any of their employees, makes any warranty, express or implied, or assumes any legal liability or responsibility for the accuracy, completeness, or usefulness of any information, apparatus, product, or process

disclosed, or represents that its use would not infringe privately owned rights. Reference herein to any specific commercial product, process, or service by trade name, trademark, manufacturer, or otherwise, does not necessarily constitute or imply its endorsement, recommendation, or favoring by the United States Government or the University of California. The views and opinions of authors expressed herein do not necessarily state or reflect those of the United States Government or the University of California, and shall not be used for advertising or product endorsement purposes. 
CHARACTERIZING AND MODELING THE APPARENT ANOMALOUS BEHAVIOR OF RESISTIVITY IN Cr-Si-O THIN FILMS

\author{
Alan F. Jankowski \\ Lawrence Livermore National Laboratory, P.O. Box 808, Livermore, CA 94550
}

The Cr-Si-O material system is of interest for use as a thin film resistor. The films are sputter deposited onto conducting substrates from metal-oxide compacts using a reactive gas mixture. The cermet films composition range from 50 to 100 vol.\% $\mathrm{SiO}_{2}$ as determined from elemental measurements of the $\mathrm{Cr}, \mathrm{Si}$ and $\mathrm{O}$ content. In a wide range of resistivities from $10^{1}$ to $10^{14} \Omega$-cm measured through the film thickness, an apparent anomalous behavior is found with the $\mathrm{Cr}$, Si and $\mathrm{O}$ composition. The anomaly can be deduced to a discontinuous variation of resistivity with film composition near $80 \mathrm{vol} . \% \mathrm{SiO}_{2}$. The film microstructure is characterized as a distribution of conducting metal-silicide particles within an insulating matrix. The "effective medium" theory is used to predict the variation of conductivity and successfully models the anomalous resistivity behavior. 


\section{INTRODUCTION}

The deposition of metal-oxide (cermet) materials is pursued in the microelectronics industry for use as resistive thin films. Cermet films with multiple phases provide a path to change the resistance by more than two-orders of magnitude. ${ }^{[1]}$ The general dependence of sheet resistance on composition was investigated for the $\mathrm{Cr}-\mathrm{Si}-\mathrm{O}$ system. ${ }^{[2-5]}$ The conduction mechanism for these cermets, as for $\mathrm{Au}-\mathrm{SiO}$ films, can be considered quantum mechanical.[6] For low metallic concentrations, charge transport is proposed to be by electron tunneling between the metallic particles.[7] In the effective medium theory, Springett considers the conductivity of a system of metallic particles dispersed in an insulating medium. In general, conduction is considered to be by means of an activated charge transport process. For film resistivities $>10^{-2} \Omega-\mathrm{cm}$, the microstructure is usually comprised of a continuous insulating matrix in which metallic particles are dispersed. The insulating matrix is based on the $\mathrm{SiO}_{2}$ phase for the $\mathrm{Cr}$-Si-O system with $\mathrm{Cr}$ and its silicides-monoxides serving as the conductors/semiconductors. A fundamental observation by Neugebauer suggests that the $\mathrm{SiO}_{2}$ composition alone can be used to determine the sheet resistance to within two orders of magnitude irrespective of deposition technique or conditions, which includes substrate heating to $600{ }^{\circ} \mathrm{C} .[1]$ This observation may not fully describe the resistance behavior of $\mathrm{Cr}-\mathrm{Si}$-O cermets. Depositions using different substrate bias conditions changed the sheet resistance by as many as five orders of magnitude for $\mathrm{Cr}-\mathrm{SiO}$ films rf-sputtered from a single composition target.[8] In addition, post-deposition annealing in a small $\mathrm{O}_{2}$ partial pressure increased the resistance as attributed to surface oxidation. ${ }^{[9]}$ Last and the present motivation, an anomalous behavior for the vertical resistance with elemental composition appeared for $\mathrm{Cr}-\mathrm{Si}$-O films in a detailed study of the resistivity behavior $>10^{2} \Omega$-cm. ${ }^{[10]}$ An attempt to model this anomalous behavior and all prior experimental results is now undertaken using the effective medium theory. This presentation provides an evaluation of both the experimental observations and fundamental postulates for the resistivity behavior of cermet films containing oxides of silicon. 


\section{EXPERIMENTAL METHOD}

The sputter deposition process transfers target chemistry in film formation. Various targets of different metallic content and oxidation states are sputtered to directly correlate changes in film resistance. Composition is measured with Rutherford Backscattering (RBS) and the film microstructure is characterized with transmission electron microscopy (TEM). The current-voltage behavior is measured through the film thickness to determine the vertical resistance which is modeled with the effective medium theory.

\subsection{Film Synthesis}

The sputter targets are fabricated from the compaction of metal and oxide powders blended by volume to the composition $(x) \mathrm{Cr}(1-\mathrm{x}) \mathrm{SiO}_{\mathrm{y}}$ (where $0.1<\mathrm{x}<0.5$ and $\left.\mathrm{y}=1 ; 2\right)$. ${ }^{[10]}$ The powder compaction methods are hot-pressed (HP) and hot-isostatic-pressed (HIP) yielding targets that are $\sim 80 \%$ and $>95 \%$ fully dense. Also, a (u) $\mathrm{Cr}(\mathrm{w}) \mathrm{Cr}_{2} \mathrm{O}_{3}(1-\mathrm{u}-\mathrm{w}) \mathrm{SiO}$ (HIP) target (where $\mathrm{u}=0.086$ and $\mathrm{v}=0.166$ ) is used to further examine the effects of metal-oxidation states. The HP method is straightforward but requires a $800-1500^{\circ} \mathrm{C}$ sinter that can result in thermal decomposition of $\mathrm{SiO}$ into $\mathrm{SiO}_{2}$. The HIP method is more complex since the powders are compacted in an evacuated container, but does avail decreased process temperature.

The film thickness (t) must be sufficient to avoid the effects seen for films thinner than $0.1 \mu \mathrm{m} .{ }^{[11-12]}$ The $0.3 \pm 0.1 \mu \mathrm{m}$ thick films are rf-sputter deposited using planar magnetrons.[10] A reactive gas mixture of $(1-\mathrm{z}) \mathrm{Ar}-(\mathrm{z}) \mathrm{O}_{2}$ (where $\left.0<\mathrm{z}<0.1\right)$ flows at $32 \mathrm{cc}-\mathrm{m}^{-1}$ for gas pressures $(\mathrm{p})$ of 1-4 Pa. The Si-wafer substrates are first sputter-coated with $0.3 \mu \mathrm{m}$ of Ni-(7wt.\%)V which serves as the bottom metal connect for the vertical resistance measurement. A rise in substrate temperature to $50-100{ }^{\circ} \mathrm{C}$ occurs during deposition. Forward powers $<3 \mathrm{~W}-\mathrm{cm}^{-2}$ are applied to the $6.3 \mathrm{~cm}$ diameter targets that are horizontally spearated $10 \mathrm{~cm}$ from the substrate. Deposition rates vary with the sputter gas pressure and target fabrication method. For example, using a $2 \mathrm{~Pa}$ Ar sputter gas, a HIP target of $0.35 \mathrm{Cr}-0.65 \mathrm{SiO}$ yields a $0.037 \mathrm{~nm}-\mathrm{W}^{-1}-\mathrm{m}^{-1}$ deposition rate whereas the HP target yields a $0.044 \mathrm{~nm}-\mathrm{W}^{-1}-\mathrm{m}^{-1}$ rate. 
The thermal stability of the microstructure and vertical resistance is accessed through a vacuum anneal. In a background pressure $<5 \times 10^{-5} \mathrm{~Pa}$, the samples are heated to $450^{\circ} \mathrm{C}$ at a $20^{\circ} \mathrm{C}-\mathrm{m}^{-1}$ rate, held for $2 \mathrm{~h}$ at temperature, then cooled at a $40^{\circ} \mathrm{C}-\mathrm{m}^{-1}$ rate to room temperature.

\subsection{Characterization of Composition and Microstructure}

The film composition (at.\%) is determined from samples deposited on ultra-densified amorphous carbon (UDAC) wafers using Rutherford Backscattering (RBS).[10] The films are analyzed using $2.3 \mathrm{MeV} \mathrm{He} \mathrm{He}^{+}$ions at normal incidence with a $4 \mathrm{~mm}^{2}$ beam spot at a $164^{\circ}$ detection angle. In addition, particle-induced x-ray emission (PIXE) spectra were collected to provide enhanced elemental specificity. The ultra-thin windowed $\mathrm{x}$-ray detector was located at a $150^{\circ}$ detection angle. The absolute number of $\mathrm{He}^{+}$ions generating each spectrum was determined by a spinning-wire dosimetry system.[13] The RBS spectra consist of well-separated signals for Cr, $\mathrm{Si}$ and $\mathrm{O}$ with essentially no background under the elemental signals. The relative counts from each of the elements is determined with high precision $(<1 \%)$ and quantifiable through the surface approximation. [14] Although the surface approximation slightly overestimates the actual number of atoms-cm ${ }^{-2}$, an accurate measure of the relative atomic concentrations $( \pm 1 \%)$ is made without requiring knowledge of the stopping cross-sections for the film. The $\mathrm{SiO}_{2}$ content (vol. $\%$ ) is determined from the $\mathrm{Cr}, \mathrm{Si}$ and $\mathrm{O}$ elemental quantities. To model the experimental data with the effective medium theory requires the assumption that $\mathrm{SiO}_{2}$ is the insulating phase. Thus, all available oxygen fully bounds $\mathrm{Si}$ as $\mathrm{SiO}_{2}$ with the remaining constituents forming the conducting particles.

Transmission electron microscopy (TEM) fully characterizes the microstructure and crystallinity. ${ }^{[10]}$ The cermet films are imaged following cross-section preparation. High resolution images are obtained near the Scherzer-defocus condition. Selected-area diffraction (SAD) patterns are used to assess crystallinity. An attempt at crystal structure determination was made using $x$-ray diffraction in which a rotating-anode source illuminated the films in the $\theta / 2 \theta$ mode. Diffuse reflections attributed to the nanometric grain size couldn't be used for phase identification from characteristic Bragg peaks. 


\subsection{Resistance Measurement}

A test method for measuring the current (i) versus voltage (V) behavior must yield a unique and reproducible resistivity value for each cermet film.[10] The resistive path through the film thickness is measured between contact pads using a semiconductor parameter analyzer. A square array $(5 \mathrm{~mm} \times 5 \mathrm{~mm})$ of four, molybdenum contact pads $(254 \mu \mathrm{m}$ diameter x $0.5 \mu \mathrm{m})$ are electron-beam deposited onto the cermet films. The i-V measurements are made from zero to 20 Volts in $200 \mathrm{mV}$ increments using all pad combinations to produce four independent measures of resistance. The resistive path is through twice the film thickness -- from the first pad to the base $\mathrm{Ni}-\mathrm{V}$ layer and back to the second surface pad assuming a negligible contribution from the metal contacts and path. The vertical resistance $(\rho)$ is determined from the field $(E)$ and current density (J) using the standard expressions

where

$$
\rho=E(J)^{-1},
$$

and

$$
\begin{aligned}
& \mathrm{E}=\mathrm{V}(2 \mathrm{t})^{-1}, \\
& \mathrm{~J}=\mathrm{I}\left(\mathrm{A}_{\mathrm{c}}\right)^{-1}
\end{aligned}
$$

noting that $\left(\mathrm{A}_{\mathrm{c}}\right)$ is the area between each contact pad and the cermet film surface.

\section{RESISTIVITY THEORY}

The conductivity for a system of metal-particles in an insulating-matrix with vol.\% metal can be modeled in terms of an effective medium theory. The theory is reviewed in brief whereas a detailed derivation can be found elsewhere. [7] The system of metal-particles in an insulatingmatrix can be considered as a lattice network of conductances. At each lattice site is associated a conductivity $\left(\sigma_{j}\right)$ whose value depends on whether the site is occupied by a metal or insulator. In a binary distribution, $\sigma_{\mathrm{m}}$ is the metallic conductivity and $\sigma_{\mathrm{i}}$ is the insulator conductivity. The occupation probability at each site is equal to $v_{j}(f)^{-1}$ where $v_{j}$ is the volume fraction and $f$ is the packing fraction. A network of lattice site probability can be considered by replacing each site 
conductance $\sigma_{\mathrm{j}}$ by an average value $\sigma^{*}{ }^{\left[{ }^{[15]}\right.}$ The normalized conductivity $(\sigma)$ is derived[7] for a cubic network (wherein $f=0.52$ for a simple cubic lattice) as

$$
\sigma=\sigma_{\mathrm{i}}\left(\sigma^{*}\right)^{-1}
$$

where

$$
\begin{gathered}
4 \sigma^{*}=\sigma_{\mathrm{i}}{ }^{\prime}+\sigma_{\mathrm{m}}{ }^{\prime}+\left[\left(\sigma_{\mathrm{i}}{ }^{\prime}+\sigma_{\mathrm{m}}{ }^{\prime}\right)^{2}+8\left(\sigma_{\mathrm{i}} \sigma_{\mathrm{m}}\right)\right]^{0.5}, \\
\sigma_{\mathrm{i}}{ }^{\prime}=\left[2-3 v_{\mathrm{m}}(\mathrm{f})^{-1}\right] \sigma_{\mathrm{i}} \\
\sigma_{\mathrm{m}}{ }^{\prime}=\left[3 v_{\mathrm{m}}(\mathrm{f})^{-1}-1\right] \sigma_{\mathrm{m}} .
\end{gathered}
$$

and

Consider again the postulate that the $\mathrm{SiO}_{2}$ composition alone can be used to determine the resistance irrespective of deposition technique or conditions. [1] The above formulation can now be used to model the resisitivity of a system of conducting-particles in an insulating-matrix with vol.\% insulator, where the resistiviy $(\rho)$ is equal to $(\sigma)^{-1}$. Two effects of $\mathrm{f}$ on $\rho$ and $\sigma$ are worth noting. As the number of nearest neighbors increases, the variation of $\rho$ and $\sigma$ with $v_{m}$ will shift to lower $v_{m}$ values. That is, resistivity decreases and conductivity increases since the neighbors are closer together. When a variety of different sized particles is considered, the packing fraction $\mathrm{f}$ will again increase. Conversely, the resistivity increases when the conducting neighbors are moved further apart.

\section{RESULTS AND ANALYSIS}

RBS measurement of the compositions verify the target values and validate the analysis procedure. For example, $43.6 \mathrm{wt} . \% \mathrm{Cr}$ is measured for the (HIP) $0.20 \mathrm{Cr}-\mathrm{SiO}$ target which is in good agreement with its design value of 45.6 wt.\% (41.8 at.\%). The elemental composition (at.\%) of several cermet films are listed in Table 1 with the computed $\mathrm{SiO}_{2}$ content (vol. \%). Impurities as the sputter gas ( $<1$ at.\% Ar) and HIP target container $(\leq 0.01$ at. $\% \mathrm{Ta})$ are found in several films. The sputtering process does not directly deposit the target composition. The film

composition depends on the gas composition whereas the effect of gas pressure appears negligible. Films deposited using a sputter gas of pure Ar are deficient in $\mathrm{Cr}$ and have an excess of $\mathrm{Si}$ and $\mathrm{O}$ relative to the target. Oxygen in the sputter gas yields a higher $\mathrm{O}$ concentration in the 
films. For example, the $\mathrm{Cr}-\mathrm{Cr}_{2} \mathrm{O}_{3}-\mathrm{SiO}$ target yields an average film composition of $10.2 \pm 0.3$ at.\% $\mathrm{Cr}$ with $37.3 \pm 0.9$ at.\% $\mathrm{Si}$ as sputtered in pure $\mathrm{Ar}$ whereas a (1-z)Ar-(z)O $\mathrm{O}_{2}$ gas composition (where $\mathrm{z}>0.03$ ) produces an average film composition of $3.9 \pm 0.4$ at.\% $\mathrm{Cr}$ with $27.7 \pm 0.6$ at.\% Si. An increase of $\mathrm{Cr}$ concentration in the film follows an increase of $\mathrm{Cr}$ content in the target. The $\mathrm{Cr}-\mathrm{Cr}_{2} \mathrm{O}_{3}-\mathrm{SiO}$ target yields the highest ratio of $\mathrm{Cr}$ in comparison to the $\mathrm{Cr}-\mathrm{SiO}$ and $\mathrm{Cr}-\mathrm{SiO}{ }_{2}$ targets. The target fabrication method does influence the composition of the deposited film. The high density (HIP) targets yield film compositions closer to the target, with more Cr and less $\mathrm{Si}$, in comparison to the low density (HP) counterparts.

The vertical resistance is computed for films of all sputter gas pressure and target combinations at a $3 \times 10^{5} \mathrm{~V}-\mathrm{cm}^{-1}$ field strength. The $\mathrm{E}$ vs $\mathrm{J}$ behavior for a typical Cr-Si-O film is shown in Fig. 1 for specimen 706. The vertical resistance measures $3 \times 10^{3} \Omega$-cm in the asdeposited condition whereas the anneal increases $\rho$ to $3 \times 10^{4} \Omega$-cm. The typical increase in $\rho$ is by a factor of 10 to 100 for films prepared using the $\mathrm{Cr}-\mathrm{SiO}$ targets. The anneal increases $\rho$ the least amount (by a factor <2) for those cermet films deposited from the $\mathrm{Cr}_{-} \mathrm{Cr}_{2} \mathrm{O}_{3}-\mathrm{SiO}$ target. Values of $\rho$ for the cermet films are listed in Table 1. In general, the cermet films become more conductive when a decrease in the $\mathrm{O}$ composition accompanies an increase in $\mathrm{Cr}$ and $\mathrm{Si}$.

TEM reveals the microstructure of the as-deposited film and the origin for the changes in $\rho$ observed upon annealing. High resolution imaging in cross-section (see Fig. 1 insert) for specimen 706 reveals the nanometric features of the microstructure. In the as-deposited region adjacent to and within $10 \mathrm{~nm}$ of the $\mathrm{Ni}-\mathrm{V}$ substrate surface, a random distribution of 1-2nm size precipitates is found within an amorphous matrix. These precipitates within the same region of the cermet have increased to 3-4 $\mathrm{nm}$ in size after the anneal. SAD yields a ring pattern for the nanoprecipitates. Interplanar spacings $(\mathrm{nm})$ of $0.21 ; 0.15 ; 0.12$ are measured which correspond with spacings (nm) of $0.204 ; 0.144 ; 0.118$ and (hkl) reflections (011); (002); (112) that match body-centered-cubic $\mathrm{Cr}$.

A general trend is confirmed that the as-deposited $\mathrm{Cr}-\mathrm{Si}-\mathrm{O}$ films are more conductive when the $\mathrm{Cr}$ composition increases in the film. ${ }^{[1-3]}$ However, anomalous variation in the resistance is observed through a continuous range of Cr-composition. ${ }^{[10]}$ The anomalous variation of 
resistance with elemental composition doesn't appear when plotted as a function of the insulator volume fraction, i.e. the vol.\% $\mathrm{SiO}_{2}$. The TEM results verify that the microstructure is best described as a distribution of conducting particles in an insulating matrix. This structure fits the assumptions of the effective medium theory. The vertical and sheet resistance measurements are plotted (in Fig. 2) for all Cr-Si-O films. ${ }^{[1-4,10]}$ A unified (qualitative and quantitative) variation of both sheet and vertical resistance with vol. $\% \mathrm{SiO}_{2}$ represents a single resistivity. Resistivity values of $10^{2}$ to $10^{3} \Omega$-cm are found over a range of 55 to 80 vol. $\% \mathrm{SiO}_{2}$ with a rapid increase to $>10^{9} \Omega$-cm for contents $>90 \mathrm{vol} . \% \mathrm{SiO}_{2}$. This discontinuity in the resistivity is well-modeled using the effective medium theory. The theoretical plot (in Fig. 2) accurately traces the experimental results asumming the conductivity ratio of particle to matrix insulator is $10^{8}$.

\section{DISCUSSION}

Cermet films are produced covering a wide range of $\mathrm{Cr}$ composition (<30 at.\%) with a corresponding $\mathrm{SiO}_{2}$ range $>55$ vol.\% using the various targets and $\mathrm{Ar}-\mathrm{O}$ gas mixtures. The effect of $\mathrm{Cr}-\mathrm{Si}-\mathrm{O}$ composition on the resistivity can be adequately assessed above $10^{0} \Omega$-cm for the films of this study. The effective medium theory is used to correctly model the resistivity $>10^{0} \Omega$-cm for the thin films in the as-deposited condition. The post-deposition anneal increases the resistance and size of the Cr-rich nanoprecipitates. The smallest $(<200 \%)$ increase in resistance is found for films deposited from the $\mathrm{Cr}-\mathrm{Cr}_{2} \mathrm{O}_{3}-\mathrm{SiO}$ target, implying it produces the most stable cermet films. The least stable films are deposited from targets with the highest fraction of free-Cr and lowest volume fraction of $\mathrm{SiO}_{2}$. The changes in resistivity for this study are not attributable to surface oxidation as is the case for the increase of sheet resistance when annealing some cermet films. ${ }^{[9]}$ The resistivity of reference metal films were invariant to the same vacuum anneal and measurement procedures. The SAD results don't eliminate the possibility that the Cr-rich nanoprecipitates have some Si content. For example, silicides were found as precipitates within an amorphous Si-O matrix for vapor deposited cermets. ${ }^{[2]}$ The growth of the precipitates may be a result of segregation of free- $\mathrm{Cr}$ from the matrix and/or the 
coalescence of the nanoprecipitates from the as-deposited condition. Particle coarsening after the anneal correlates with an increase of nearest neighbor spacing, hence an increase in $\rho$ as predicated in the final remarks of Sec. 3 for the effective medium theory. In addition, the conductivity of the Cr-rich nanoprecipitates may decrease consequent to growth as a silicide.

The resistivity of annealed $\mathrm{Cr}-\mathrm{Si}-\mathrm{O}$ films prepared by flash-evaporation decreased as attributed to an extended clustering of Cr-rich (silicide) precipitates. [2] The silicide precipitates became interconnected as a result of the anneal treatments forming a near continuous web through the matrix. The distribution of precipitates subsequent to annealing for film compositions $>55$ vol. $\% \mathrm{SiO}_{2}$ in this study aren't described by continuous cluster coalescence. A semi-conductor to insulator transition is consistent with the microstructure observed (in Fig. 1) for metal particles in an insulating matrix and the range of resistivity data $>10^{0} \Omega$-cm (in Fig. 2). For resistivities $<10^{0} \Omega-\mathrm{cm}$, a different microstructure may be anticipated in which the metal phase is nearly continuous constituting a conducting to semi-conducting transition. The effective medium theory proposed by Springett does not rigorosuly deal with a near continuous conducting phase. However, the approach by Bueche does assume a near continuous conducting phase. ${ }^{[16]}$ The variation of conductivity with volume fraction metal are very similar for both microstructures. A more abrupt transition for $\rho$ vs vol. $\% \mathrm{SiO}_{2}$ results when conductance is modeled as dependent on only those metallic particles which form a connected chain through the sample. In both approaches, the average conductance is formed between the number of particles or chains and the remaining matrix. With respect to the present findings, three resistivity plateaus may then be found corresponding to a conductor, semi-conductor and insulator. Resistivity in the Cr-Si-O system is highly dependent on the morphology and distribution of the conducting phase. 


\section{SUMMARY}

Sputtering was used to deposit thin film resistors from various Cr-Si-O cermet targets. ${ }^{[10]}$ Measurements through the resistor thickness show an increase in $\rho$ from $10^{2} \Omega$-cm for films deposited using pure Ar to values $>10^{8} \Omega$-cm for depositions using an Ar-O gas mixture. The microstructure was shown to be composed of $\mathrm{Cr}$-rich nanoprecipitates within an insulating silica matrix for compositions $>55$ vol. $\% \mathrm{SiO}_{2}$. The as-deposited microstructure and resistivity behavior were unstable upon a vacuum anneal. The $\mathrm{Cr}-(\mathrm{Si})$ precipitates increased in size. The resistivity increased by factors of 10 to 100 for films prepared using $\mathrm{Cr}-\mathrm{SiO}$ and $\mathrm{Cr}-\mathrm{SiO}_{2}$ targets whereas deposits from the $\mathrm{Cr}-\mathrm{Cr}_{2} \mathrm{O}_{3}-\mathrm{SiO}$ target were most stable. The effective medium theory proposed by Springett[7] is used to model the resistivity behavior, not as function of elemental composition(s) but as a function of volume fraction $\mathrm{SiO}_{2}$. An abrupt and apparently anomalous increase in the resistivity at $80 \mathrm{vol} \% \mathrm{SiO}_{2}$ is accounted for using the model. An observation by Neugebauer ${ }^{[1]}$ suggesting that the $\mathrm{SiO}_{2}$ composition alone can be used to determine the cermet resistance to within two orders of magnitude irrespective of deposition technique or conditions which include substrate heating, appears vaild with provision for an additional stipulation that the variation is not continuous but depends on the distribution and morphology of the conducting phase.

\section{Acknowledgment}

This work was performed under the auspices of the United States Department of Energy by Lawrence Livermore National Laboratory under contract \#W-7405-Eng-48. 


\section{References}

1. C. A. Neugebauer, Thin Solid Films, 6 (1970) 443.

2. R. Glang, R. A. Holmwood and S. R. Herd, J. Vac. Sci. Technol., 4 (1967) 163.

3. A. A. Milgram and C-S. Lu, J. Appl. Phys., 39 (1968) 4219.

4. $\quad$ N. C. Miller and G. A. Shirn, Solid State Tech., 11 (1968) 28.

5. H. Steemers and R. Weisfield, Mater. Res. Soc. Symp. Proc., 118 (1988) 445.

6. J. E. Morris, Thin Solid Films, 11 (1972) 299.

7. B. E. Springett, J. Appl. Phys., 44 (1973) 2925.

8. H. Matino and T. Ushiroda, IBM J. Res. Develop., 21 (11) (1977) 576.

9. A. D. Katnani, L. J. Matienzo and F. Emmi, Thin Solid Films, 204 (1991) 265.

10. A.F. Jankowski, J.P. Hayes, R.G. Musket, F. Cosandey, C.E. Gorla, R.S. Besser, V. Westerlind and G. Cobai, in E. Ma, B. Fultz, R. Schull, J. Morral and P. Nash (eds.), Chemistry and Physics of Nanostructures and Related Non-Equilibrium Materials, TMS Symposia Proceedings, Metals Park, 1997, p. 211.

11. T. Filutowicz, W. Gregorczyk and B. Stepien, Electron Technology, 10 (1977) 117.

12. H. S. Hoffman and E. Stephans, IEEE Trans. on Components, Hybrids and Manufacturing Technol., 4 (4) (1981) 387.

13. R. G. Musket, R. S. Daley and R. G. Patterson, Nucl. Instr. and Meth., B83 (1993) 425.

14. RUMP program, Computer Graphics Service, Lansing, NY 14882, USA.

15. S. Kirkpatrick, Phys. Rev. Lett., 27 (1971) 1722.

16. F. Bueche, J. Appl. Phys., 43 (1972) 4837. 
Table I. Composition and Resistance of Sputter-Deposited Cr-Si-O Films

\begin{tabular}{|c|c|c|c|c|c|c|c|c|c|}
\hline Sample & Target & $\mathrm{p}(\mathrm{Pa})$ & $(\mathrm{z})\left(\mathrm{O}_{2}\right)$ & $\mathrm{Cr}($ at.\%) & $\mathrm{Si}($ at.\%) & $\mathrm{O}($ at.\%) & $\operatorname{Ar}($ at. \%) & $\mathrm{SiO}_{2}($ vol. \%) & $\rho(\Omega-\mathrm{cm})$ \\
\hline 706 & $0.20 \mathrm{Cr}-0.80 \mathrm{SiO}(\mathrm{HP})$ & 2 & 0.000 & 9.9 & 34.9 & 54.9 & 0.3 & 82.4 & $3.0 \times 10^{3}$ \\
\hline 726 & $0.20 \mathrm{Cr}-0.80 \mathrm{SiO}(\mathrm{HIP})$ & 1 & 0.000 & & & & & & $8.0 \times 10^{2}$ \\
\hline 623 & $0.20 \mathrm{Cr}-0.80 \mathrm{SiO}(\mathrm{HIP})$ & 2 & 0.000 & & & & & 81.9 & $1.0 \times 10^{2}$ \\
\hline target & $0.20 \mathrm{Cr}-0.80 \mathrm{SiO}(\mathrm{HIP})$ & - & - & & 29.1 & & 0.0 & - & - \\
\hline 811 & $0.35 \mathrm{Cr}-0.65 \mathrm{SiO}(\mathrm{HP})$ & 4 & 0.000 & 24.1 & 36.9 & 38.1 & 0.0 & 57.3 & $8.0 \times 10^{2}$ \\
\hline 701 & $0.35 \mathrm{Cr}-0.65 \mathrm{SiO}(\mathrm{HP})$ & 2 & 0.000 & & .5 & & 0.3 & 6 & $5.0 \times 10^{1}$ \\
\hline 707 & $0.35 \mathrm{Cr}-0.65 \mathrm{SiO}(\mathrm{HIP})$ & 2 & 0.000 & 25.7 & 28.6 & & 0.4 & 0.8 & $2.0 \times 10^{3}$ \\
\hline 913 & $0.35 \mathrm{Cr}-0.65 \mathrm{SiO}(\mathrm{HIP})$ & 2 & 0.033 & & & & & 94.9 & $3.0 \times 10^{10}$ \\
\hline target & $0.35 \mathrm{Cr}-0.65 \mathrm{SiO}(\mathrm{HIP})$ & - & 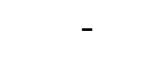 & 60.6 & 19.7 & 19.7 & 0.0 & - & - \\
\hline 608 & $0.35 \mathrm{Cr}-0.65 \mathrm{SiO}_{2}(\mathrm{HP})$ & 2 & 0.000 & 13.3 & 24.5 & 62.2 & 0.0 & 87.5 & $3.0 \times 10^{8}$ \\
\hline 630 & $0.35 \mathrm{Cr}-0.65 \mathrm{SiO}_{2}(\mathrm{HIP})$ & 2 & 0.000 & 23.5 & 20.0 & 56.0 & 0.5 & 76.6 & $0.0 \times 10^{3}$ \\
\hline target & $0.35 \mathrm{Cr}-0.65 \mathrm{SiO}_{2}(\mathrm{HP})$ & - & 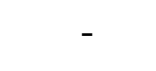 & 67.8 & 10.7 & 21.5 & 0.0 & - & - \\
\hline 1012 & $\mathrm{Cr}-\mathrm{Cr}_{2} \mathrm{O}_{3}-\mathrm{SiO}(\mathrm{HIP})$ & 2 & 0.000 & 10.0 & 39.3 & 49.3 & 1.4 & 73.1 & $1.0 \times 10^{3}$ \\
\hline 1019 & $\mathrm{Cr}-\mathrm{Cr}_{2} \mathrm{O}_{3}-\mathrm{SiO}(\mathrm{HIP})$ & 2 & 0.066 & 4.7 & 27.5 & 67.2 & 0.6 & 95.7 & $1.3 \times 10^{13}$ \\
\hline target & $\mathrm{Cr}-\mathrm{Cr}_{2} \mathrm{O}_{3}-\mathrm{SiO}(\mathrm{HIP})$ & - & - & 26.4 & 33.6 & 40.0 & 0.0 & - & - \\
\hline
\end{tabular}




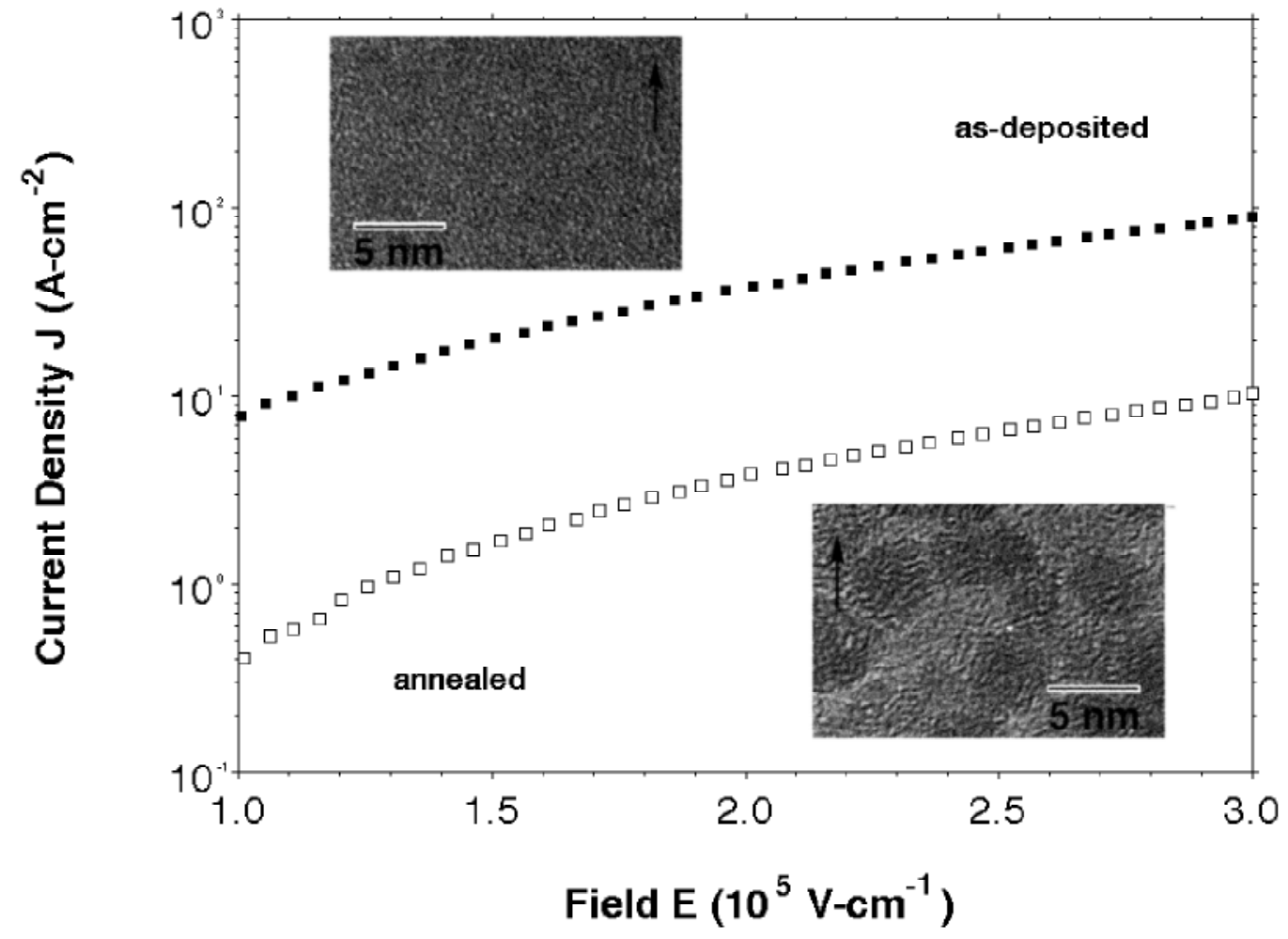

Figure 1.

A plot of the field $\left(\mathrm{V}-\mathrm{cm}^{-1}\right)$ versus current density $\left(\mathrm{A}-\mathrm{cm}^{-2}\right)$ of a $\mathrm{Cr}-\mathrm{Si}-\mathrm{O}$ cermet film in the asdeposited and annealed condition with high resolution images (insert). [10] The arrow indicates the growth direction and the marker is $3 \mathrm{~nm}$ in length. 


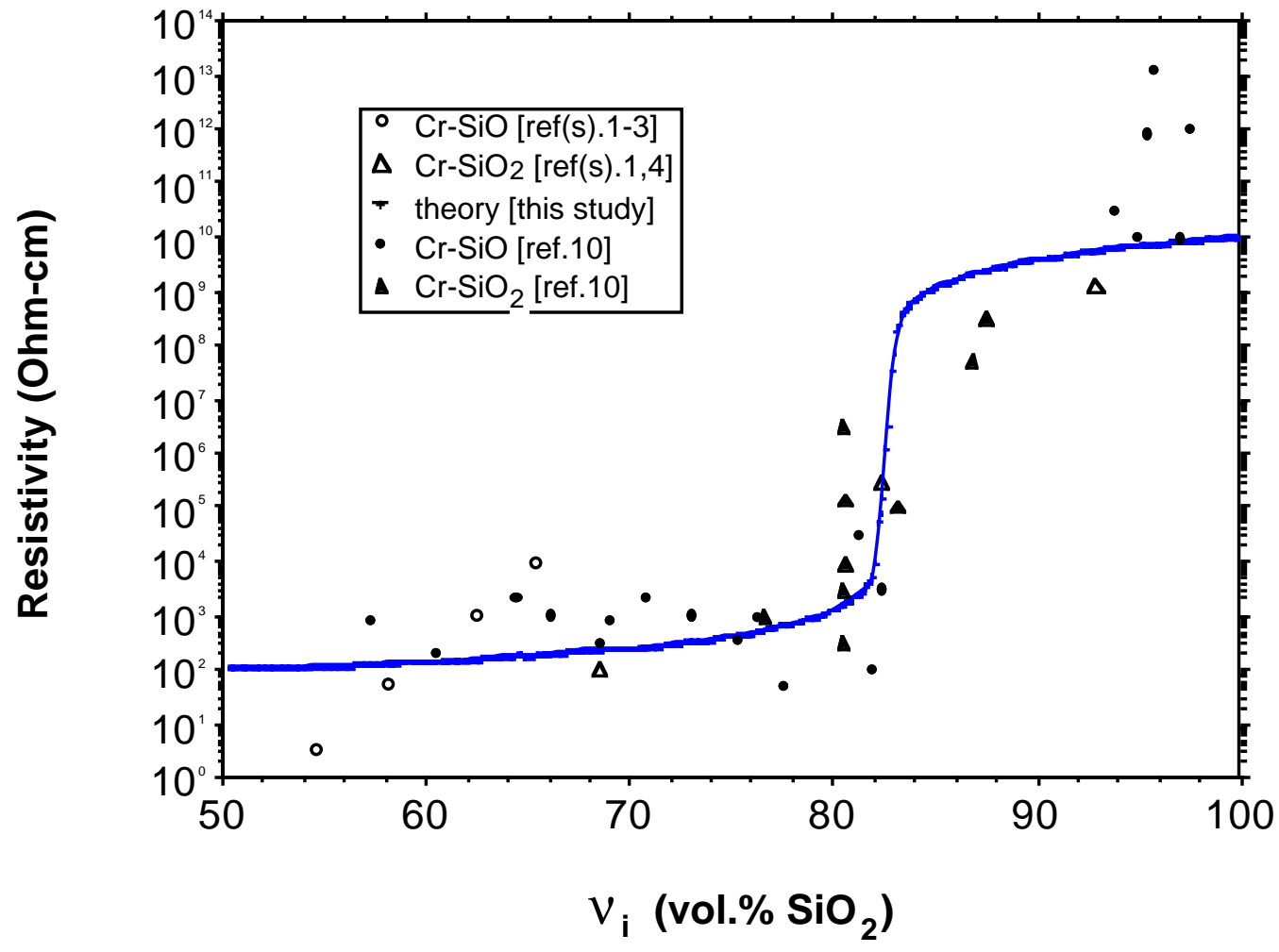

Figure 2.

A plot of the insulator composition (vol.\% $\left.\mathrm{SiO}_{2}\right)$ versus resistivity $(\Omega-\mathrm{cm})$ as experimentally measured and modeled for $\mathrm{Cr}-\mathrm{Si}-\mathrm{O}$ films. 


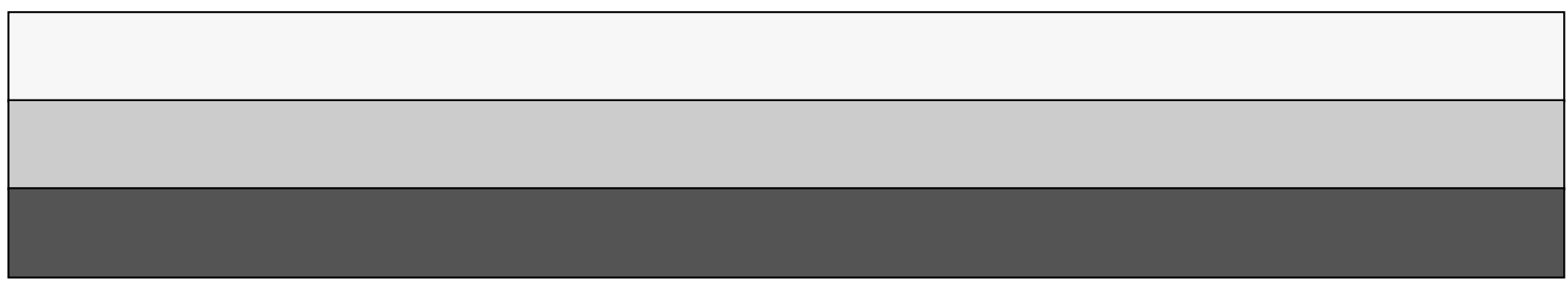

